\title{
TEST RESULTS FOR AN OFF THE SHELF GPS/INS DURING APPROACH AND LANDING TESTING OF THE X-40A
}

\author{
Dave Childers, Boeing Reusable Space Systems, Seal Beach, Califormia \\ Hendrik Gelderloos, Honeywell Space Systems, Clearwater, Florida
}

\begin{abstract}
The X-37 is an unpiloted, reusable space vehicle that will be launched into space, orbit the earth, reenter the atmosphere and land autonomously. At the heart of the Guidance, Navigation and Control (GN\&C) will be the Space Integrated GPS/INS (SIGI) system, an off the shelf navigation grade GPS/INS that has been enhanced for space and reentry environments. SIGI will provide both navigation and flight control data to the X-37's GN\&C.
\end{abstract}

The $\mathrm{X}-40 \mathrm{~A}$ is an unpiloted experimental vehicle whose shape and performance are similar to the X-37's and was flown earlier this year to develop and test the approach and landing phase of $\mathrm{X}-37$. On board the X-40A is the X-37's SIGI, which is riding along as an experiment. The $\mathrm{X}-40 \mathrm{~A}$ SIGI experiment provided early characterization of SIGI operation and performance with differential GPS during real world approach and landing. Characterization testing was geared toward assessment of reliability and performance of the system. The objectives were to demonstrate performance levels sufficient to meet the X-37 requirements for automatic, autonomous approach and landing, demonstrate reliability over repeated ground and flight tests and reduce risk for integration of SIGI into the vehicle and support environment. This paper presents a summary of this testing and the results to date.

\section{Introduction}

The X-37 technology demonstrator is a reusable space plane being built by Boeing Phantom Works at Seal Beach, Ca. The X-37 is designed to be carried into space on the shuttle, or an expendable booster, such as the Delta IV, and initialize itself on orbit. Once on orbit, it will perform various operations, deorbit upon ground command, reenter the earth's atmosphere and land autonomously at a designated landing site. The $\mathrm{X}-37$ is designed to demonstrate technologies in the orbital and reentry environments for nextgeneration reusable launch vehicles. The technologies it will be demonstrating include autonomous guidance and control of a low $L / D$ vehicle for which reliable and accurate navigation is essential.

The program requirements included maximization of autonomy, especially for in space initialization and reentry navigation, and included a planned mission to perform satellite rendezvous using relative GPS navigation. These requirements dictated the use of a GPS aided navigation system. Other requirements included the potential to operate for long periods without aiding and an all attitude capability. These meant that a navigation grade inertial system $(<1 \mathrm{Nm} / \mathrm{hr})$ was required. Finally, the precision required for the vehicle's autonomous landing meant that a differential GPS (dGPS) receiver was needed.

While Boeing has the expertise and experience to design and build a GPS aided inertial navigation system to meet the X-37's requirements, the pace of the program did not give time to develop such a system. Trade study conclusions were that procuring an off the shelf, aircraft type, INS/GPS, which was, or could easily be, modified for space use would be the best design. The major advantage to this was that much of the development and qualification would have been completed with only minor additional effort and time needed to adapt it to the X-37's mission. Also, Boeing recently had experience with this type of design on the $\mathrm{X}-40$ approach and landing test vehicle, discussed below.

Honeywell International (HI), Commercial Space Operations CSO had developed a navigation grade, GPS aided, inertial navigation system modified for use in space vehicles called the Space Integrated GPS/INS (SIGI). The SIGI is based on HI's H-764G Embedded GPS/INS (EGI), which is used on several military aircraft systems, including the F-14, F-16 fighters and the C-17 transport. It 
has hardware and software modifications for use by space applications. For example it incorporates radiation hardened RAM and an improved navigation mechanization for orbital dynamics. The SIGI had been developed for several space applications, one of which is as a possible replacement of the space shuttle's IMU and GPS navigation subsystems. As part of that development it has flown in space many times as a stand alone experiment in the space shuttle. The shuttle version is called the STS-SIGI (STS for Space Transportation System). The X-37-SIGI shares much heritage of the STS-SIGI, but it differs from the STS-SIGI in at least one important way: it uses a Trimble Force 512 channel receiver instead of the Collins 5 channel GEM-III. The Force 5 used for $\mathrm{X}-37$ is a dGPS capable Standard Positioning Service (SPS) receiver modified for use in space.

The X-40A approach and landing test (ALT) vehicle is similar to the $\mathrm{X}-37$, but scaled down by about $85 \%$. It is the same vehicle as was used by Boeing in August 1998 to demonstrate successful autonomous landing of a low $L / D$ vehicle. Several modifications were incorporated into the $\mathrm{X}-40$ in order to make it a test vehicle for the X-37 program. One of the major purposes of X-40A was to demonstrate the GN\&C approach and landing algorithms that will be used on X-37. Another purpose was to provide a test bed on which to host experiments for X-37. A prototype X-37-SIGI was flown on board the $\mathrm{X}-40 \mathrm{~A}$ as an experiment to characterize SIGI performance and reliability for the $\mathrm{X}-37$ program. The test objectives for the $X-40$ A flowed out of the lessons learned from the 1998 X-40 ALT program and were meant to reduce the risk to the $\mathrm{X}-37$ program.

\section{Test Objectives of SIGI Experiment}

The purpose of flying the SIGI on the X-40A was to gain experience with the system and to characterize performance and reliability early in the program. Because of the fast pace of X-37 and the critical nature of navigation in the vehicle, it was important to test the SIGI as soon and realistically as possible. The $\mathrm{X}-40 \mathrm{~A}$ provided the vehicle to do this.

The performance of the SIGI was not expected to be surprising. For the X-40A experiment it would be operating in a terrestrial aircraft environment, for which the H-764 EGI was designed and has been successful. The purpose of the $\mathrm{X}-40 \mathrm{~A}$ flight testing was to verify the SIGI's performance and reliability was not impacted by the modifications required to make navigation in space possible. (1)

The objectives of the X-40A SIGI experiment were to provide a real world, approach and landing trajectory under operational conditions in which to:

1. Understand and master the SIGI interface and operation, especially initialization and dGPS.

2. Characterize SIGI and Force 5 reliability

3. Characterize SIGI navigation performance

4. Characterize Force 5 GPS performance

5. Provide data to develop and validate HWIL simulation models

6. Provide hardware and software lessons learned, which can be incorporated into the $\mathrm{X}-37-$ SIGI and $\mathrm{X}-37$ design

These objectives were intended to get the X-37 SIGI integration and validation effort off the ground quickly. They also would allow identification of any changes in the SIGI design itself while such changes were still possible and affordable.

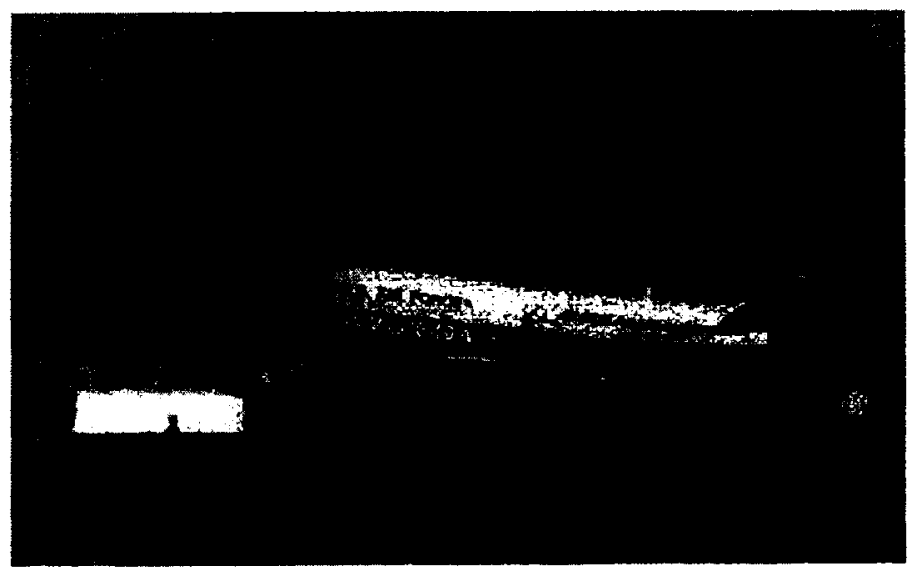

Figure 1. X-40A: Another Successful Landing

\section{Test Setup}

\section{Vehicle Configuration}

The X-40A vehicle resembles a conventional aircraft except that its wings are unusually short and the normal elevator and vertical stabilizer aero surfaces are not present. Instead the vehicle uses a 
pair of "ruddervators", tail surfaces at about a 45 deg inclination to the body, coordinated with wing flaps for flight control (see figure 1). The shortened wings are those required of a space reentry vehicle and give rise to the vehicle's distinctive low lift over drag ratio $(\mathrm{L} / \mathrm{D})$. The $\mathrm{X}-40 \mathrm{~A}$ is unpowered, which combined with the low $\mathrm{L} / \mathrm{D}$ provides a guidance and control challenge not present in other autonomous landing vehicles.

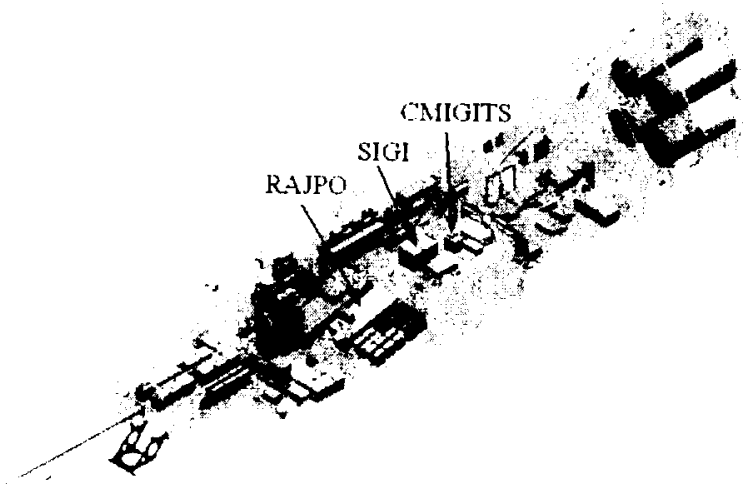

Figure 2. $X-40 A$ internal view showing avionics layout

The $\mathrm{X}-40 \mathrm{~A}$ has a large internal bay that is separated down the middle by a keel into two compartments. Batteries, avionics systems and a range safety truth source take much of the bay up. The vehicle uses a Boeing built CMIGITS, a tactical grade INS/dGPS for navigation. The CMIGITS uses a SPS GPS receiver and dGPS corrections. The Boeing designed software resident in the flight computer performs guidance, control and auxiliary navigation functions. A separate computer called the Experiment Controller (EC) was used to interface to the $\mathrm{X}-40 \mathrm{~A}$ experiments, including SIGI. The EC was completely independent from the flight computer and vehicle flight control.

The truth reference Time Space Position Information (TSPI) data was supplied by the Range Applications Joint Program Office (RAJPO) Advanced Range Data System (ARDS) using a keyed, dual frequency, differentially corrected GPS/INS on board the vehicle. The ARDS will be referred to as the "RAJPO unit", because that was the custom of the flight crew during testing. The
RAJPO, CMIGITS and the SIGI were all located on the starboard side near the center of gravity of the vehicle and within approximately 1-2 feet of each other, as shown in figure 2 . Their exact coordinates were surveyed after installation using a laser tracker device whose measurement error is on the order of 0.060 in.

Table 1. SIGI IMU Error Characterization

\begin{tabular}{|c|c|c|}
\hline Parameter & Units & $\begin{array}{l}\text { Accuracy } \\
(10)\end{array}$ \\
\hline \multicolumn{3}{|l|}{ Gyro } \\
\hline - Bias & $\mathrm{deg} / \mathrm{hr}$ & 0.004 \\
\hline - $\quad$ Angular Random Walk & $\operatorname{deg} / V_{\mathrm{hr}}$ & 0.0025 \\
\hline - Scale Factor & $\mathrm{ppm}$ & 2 \\
\hline - IA Nonorthogonality & arc-sec & 3 \\
\hline \multicolumn{3}{|l|}{ Accelerometer } \\
\hline - Bias & $\mu \mathrm{g}$ & 30 \\
\hline - $\quad$ Scale Factor & ppm & 40 \\
\hline - Scale Factor Asymmetry & ppm & 20 \\
\hline - Non-linearity & $\mu \mathrm{g} / \mathrm{g}^{2}$ & 20 \\
\hline - IA Nonorthogonality & arc-sec & 5 \\
\hline $\begin{array}{l}\text { Gyro/Accelerometer IAs to } \\
\text { Chassis Alignment }\end{array}$ & $\operatorname{arc}-\sec$ & 60 \\
\hline $\begin{array}{l}\text { INS/GPS Chassis installation } \\
\text { alignment repeatability }\end{array}$ & Arc-sec & 36 \\
\hline
\end{tabular}

\section{SIGI Configuration}

The SIGI, shown in figure 3 , is a tightly coupled GPS aided strapdown INS using a navigation grade IMU (CEP rate $<0.8 \mathrm{nmi} / \mathrm{hr}$, See Table 1 ) and a Trimble SPS Force 5 "all in view" 12 channel GPS receiver with space/orbital modifications. Both blended and GPS solutions are available.

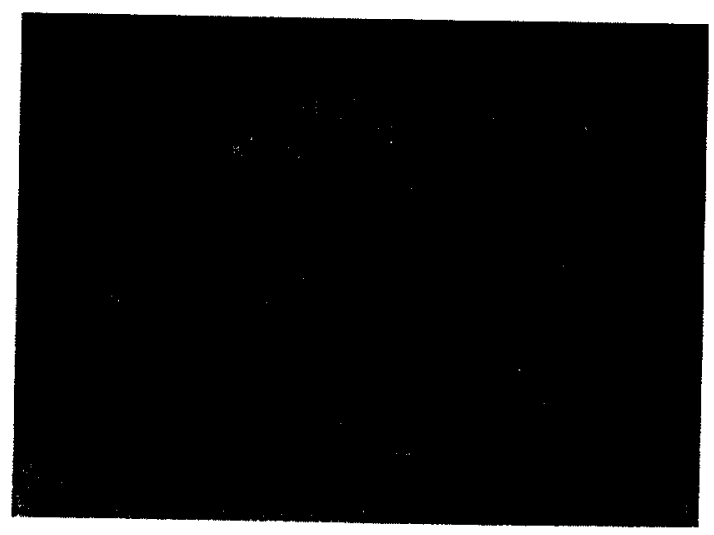

Figure 3. X-37 SIGI 
The SIGI blended GPS/INS solution uses a 29 state Kalman filter for GPS measurement incorporation. When not using differentially corrected GPS measurements, the X-37 SIGI Kalman filter measurement error uncertainties are based on Selective Availability (SA) SPS performance.

The X-37 SIGI will provide navigation state (time, position and velocity) and attitude data via its 1553 bus, and high frequency body attitude rates and linear acceleration for vehicle flight control via an RS-422 interface. Blended navigation state and attitude data are available in geodetic latitude, longitude, MSL and ellipsoid altitude, North, East, Down (NED) velocity, and ECEF frame, as well as J2000 Earth Centered Inertial (ECI) frame.

The Force 5 GPS receiver to be used for X-37 will include software modified for orbital operations. These modifications include a $\mathrm{J} 2$ gravity model for ballistic propagation during times in which GPS RF and inertial aiding is unavailable, and increased altitude and velocity limits to permit operation at orbital altitudes and speed.

Table2. comparison of SIGI versions from CDR

\begin{tabular}{|c|c|c|c|c|c|c|c|}
\hline & \multicolumn{4}{|c|}{ Hardware } & \multicolumn{4}{c|}{ Software } \\
\cline { 2 - 8 } & $\begin{array}{c}\text { RAD } \\
\text { Hard } \\
\text { SRAM }\end{array}$ & $\begin{array}{c}\text { Power } \\
\text { Supply }\end{array}$ & $\begin{array}{c}\text { I PPS } \\
\text { Certifd } \\
\text { Board }\end{array}$ & $\begin{array}{c}\text { Orbital } \\
\text { Up- } \\
\text { grades }\end{array}$ & $\begin{array}{c}\text { INS- } \\
\text { only, } \\
\text { ECI } \\
\text { Frame }\end{array}$ & $\begin{array}{c}\text { Forc } \\
\text { e 5 } \\
\text { SEU } \\
\text { Mod }\end{array}$ & $\begin{array}{c}\text { Force } \\
5 \text { S/W }\end{array}$ \\
\hline $\begin{array}{c}\text { Flight } \\
\text { Units }\end{array}$ & Yes & $\begin{array}{c}\text { Up- } \\
\text { graded }\end{array}$ & Yes & Yes & Yes & Yes & 1.60 \\
\hline $\begin{array}{l}\text { X- } \\
\text { 40A }\end{array}$ & No & $\begin{array}{c}\text { Com- } \\
\text { mercial }\end{array}$ & N/A & Yes & No & No & 1.59 \\
\hline
\end{tabular}

The prototype SIGI available for X-40A

testing was essentially an off the shelf unit that included most of the significant hardware to be used in the X-37. It did not include all of the X-37 software, which was being developed while the $X$ 40A flight test program was ongoing. Specifically, it did not include the software for the ECI coordinate reference frame, an absolute time tag, nor the hardware for the RS-422 flight control data. It did, however, include the latest space rated Force 5 software that is planned to be used in the X-37 SIGI. Table 2 summarizes the differences between the tested and the X-37 SIGIs.

\section{Ground Support}

Ground support was located in the Field Operational Control Center (FOCC), a mobile trailer unit build by Boeing for the X-40A and X-37 flight test program. The FOCC is the primary center for control and monitoring of the test. It sends commands to the vehicle and receives telemetry data where it is displayed in real time for the test conductors. The telemetry included 50,10 and $1 \mathrm{~Hz}$ data from the SIGI. The telemetry data was collected and archived at the FOCC for playback and data set post processing after each test.
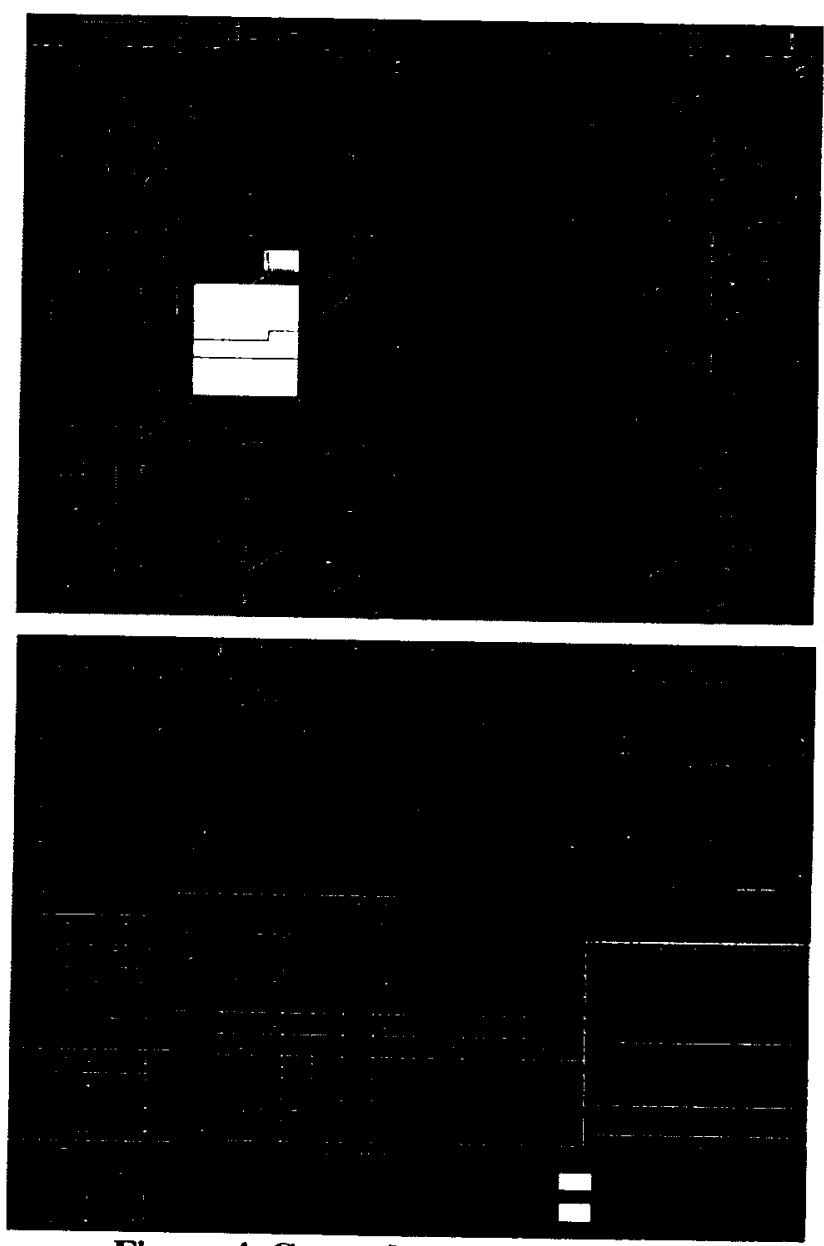

Figure 4. Ground and Drop Display

Special displays were developed to monitor SIGI performance. Two displays were used for SIGI flight test monitoring: a low bandwidth display for the drop phase of testing and a higher bandwidth display with more detailed data for troubleshooting problems while the vehicle was on the ground. (See Figure 4) 
The drop display included just the information required to assess SIGI performance and make go/no go decisions. This included relative position, velocity and attitude differences between the SIGI and RAJPO, GPS tracking and measurement incorporation, use of dGPS corrections and the RMS of Kalman filter residuals. The SIGI's operation was good to go when these parameters were all within expected limits and ranges.

The ground display included additional details such as individual GPS channel status, navigation state error plots and the Kalman filter residuals. The ground display became useful for the test program, because it also contained a GPS satellite vehicle (SV) azimuth-elevation sky plot, which helped identify times in which the CMIGITS GPS receiver might have problems due to SV obscuration (due to the helicopter or strong back, mostly).

The data in the displays were processed real time from the telemetry link with the vehicle. The three navigation systems operated asynchronously, therefore the data was referenced to a common time point by interpolation or extrapolation in order to provide real time estimates of the relative navigation errors. For the RAJPO and CMIGITS units this was straight forward, because their navigation states are time tagged with GPS time or UTC. For SIGI this was a bit of an issue, because the blended navigation solution was not time tagged using an absolute time. Specifically, the blended navigation used the 1553 mode code synch option for message time tagging which generates a $64 \mu \mathrm{sec}$ least significant bit time tag relative to the host's input synch pulse. This time tagging technique is generally applicable to vehicles with a master time source. The X-40A did not use this feature, therefore the SIGI blended solution time tag was free running.

The ground and post processing software handled the time tagging for the SIGl's blended solution by using a time offset derived from other SIGI data. The ground processing time tag was adequate for flight test monitoring, but was not successful for performance. This issue will not apply to the X-37 SIGI, because HI is adding a GPS time tag (week and seconds) to the navigation solution messages making comparisons with other navigation sources straightforward. The GPS time tag software was not available in time for the X40A SIGI flight test program.

The dGPS reference corrections were provided by an Ashtech Z-XII 12 channel GPS receiver base station broadcasting type 1 and 2 corrections every 3 seconds. The Ashtech receiver was in the FOCC and had its antenna located on top a nearby hanger. The antenna location was surveyed by NIMA at Edwards to centimeter accuracy.

The RAJPO INS/GPS truth reference used an independent source of dGPS corrections supplied by the range.

\section{SIGI Integration}

The SIGI physical location in the X-40A vehicle is shown in figure 2 . The SIGI was hard mounted to the aluminum structure inside the vehicle's avionics payload bay. This provided a rigid mounting surface for the unit that was common to all three navigation systems.

The SIGI was mounted so that its chassis coordinate frame was co-aligned with the $\mathrm{X}-40 \mathrm{~A}$ vehicle frame. Boresighting the SIGI was performed in only a crude manner from the laser tracker survey. The precision mounting feet of the SIGI were not available to sight in, nor was a tool available to survey the mounting surface. Therefore, the SIGI chassis corners were sighted in to give a rough orientation relative to vehicle coordinates. The boresight values for the SIGI were small enough for test purposes that they were not input to the unit. Data was collected from all three navigation units to determine the relative alignment and was verified to agree with the crude boresight data.

All commands and data in and out of the SIGI where handled by the EC. The software interface was through the SIGI's 1553 input and output. The SIGI configuration includes a generous supply of 1553 output messages. The $\mathrm{EC}$ was programmed to monitor thirteen different 1553 messages from the SIGI and pass over 300 data words at various rates to telemetry for display and archival on the ground by the FOCC. Table 3 provides a list of the messages monitored and their useful data.

Table 3. Monitored SIGI Messages 


\begin{tabular}{|c|l|l|}
\hline No. & \multicolumn{1}{|c|}{ Message } & \multicolumn{1}{c|}{ Comments } \\
\hline 1 & GPS Initialization & Nav mode word \\
\hline 2 & GPS Init Output & GPS init confirm \\
\hline 3 & Diff GPS, type 1 & dGPS recd conf \\
\hline 4 & GRAM LOS Data & dGPS Confirm \\
\hline 5 & SV almanac & For SV health \\
\hline 6 & GPS 1 Hz geodetic soln & GPS geodetic \\
\hline 7 & Kalman State \& Covar & State \& cov est. \\
\hline 8 & Kalman filter resid & Resid \& chi sa \\
\hline 9 & Blended 10 Hz geodic & Monitor real time \\
\hline 10 & Time Mark & UTC, ECEF \\
\hline 11 & GPS Status & Channel track \\
\hline 12 & Subsystem Status & Subsystem Fail \\
\hline 13 & INS only geodetic soln & Init align conf \\
\hline
\end{tabular}

A key element of the EC Software was to provide the type 1 differential GPS corrections to the SIGI over the 1553. Typically this data is supplied from the onboard dGPS radio receiver and sent directly to the embedded GPS receiver in RTCM-104 format via an RS-232 serial line. The Force 5 SIGI design required that the dGPS data be supplied to the SIGI in the GRAM format as a 1553 input message. Since the same design for dGPS will be used for X-37, this situation provided an opportunity to validate the dGPS SIGI interface on the X-40A program.

\section{Test Description}

The X-40A test program culminated in seven successful landings of the vehicle after being released from an Army CH-47D Chinook helicopter at $15,000 \mathrm{ft}$ above ground level. The test program was performed in a build up fashion starting from static integration testing to the helicopter drops. The testing of importance to SIGI consisted of a series of static tests, followed by taxi tow tests at increasing speed, helicopter captive carries and finally the approach and landing drop tests. SIGI data was collected for three static tests, ten taxi tow tests, eight captive carry test and seven approach and landing drops.

The static testing was performed on the vehicle and was designed to test the vehicle's interfaces with the SIGI, SIGI initialization, telemetry and displays. SIGI initialization was performed using a laptop with a 1553 card and special software. This required that the $\mathrm{X}-40 \mathrm{~A}$ 's avionics bay door be opened for access to the SIGI and EC. The laptop was connected to the experiment 1553 bus at the start of each test sequence and used to mode the SIGI and provided appropriate initialization data. Once the SIGI completed alignment and was verified to be performing correctly, the laptop was removed and the $\mathrm{X}-40 \mathrm{~A}$ avionics bay door closed.

The taxi tests consisted of towing the $\mathrm{X}-40 \mathrm{~A}$ behind a truck on the runway at speeds of up to 60 $\mathrm{mph}$ to validate the final phase of rollout. This data was also used to verify that GPS and differential GPS broadcast messages could be received with no interference at the landing site.

For the flight tests the $\mathrm{X}-40 \mathrm{~A}$ was carried airborne about 100 feet underneath a $\mathrm{CH}-47 \mathrm{D}$ helicopter attached to a tether strap. The tether was attached to a rigid structure (strong back) designed to transfer the load evenly to the vehicle. A small parachute dragged behind it stabilized the vehicle.
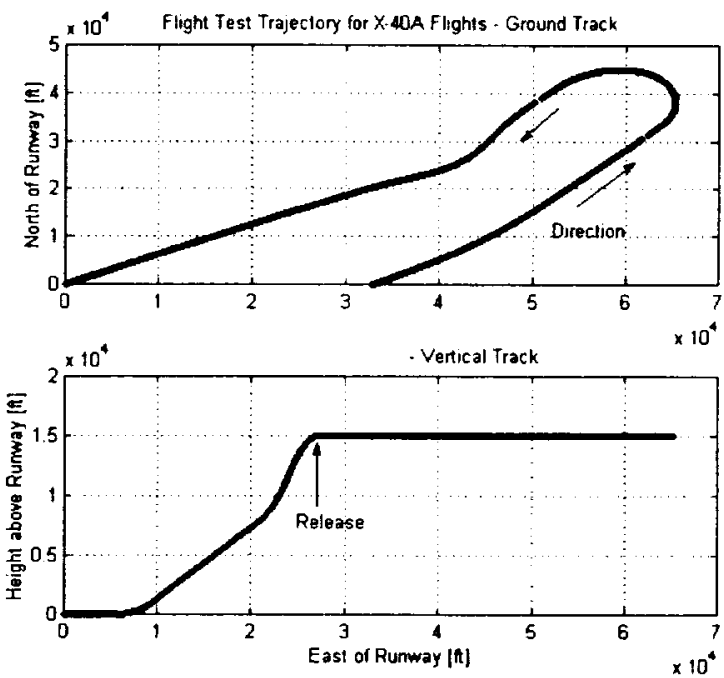

Figure 5. X-40A Pre-release and drop trajectory

The flight test sequence consisted of climbing to altitude and maneuvering the vehicle into position for release. The captive carry sequences consisted of several dry passes to the release window. Between each dry pass the helicopter would circle around in a "racetrack" oval pattern to line up for the next pass. (See figure 5) During the dry passes the GPS SV tracking and differential GPS message reception was evaluated to ensure that it was working nominally. Attitude alignment was improved by the GPS measurements along with the trajectory of the vehicle up to altitude and around the oval. 
The drop tests started with a release from $15,000 \mathrm{ft} \mathrm{AGL}$ at $225 \mathrm{ft} / \mathrm{sec}$ and the vehicle's initial heading into the runway. The flight profile produced a flight time of about 70 seconds from release to touchdown. Maximum speed was about $530 \mathrm{ft} / \mathrm{sec}$ (Mach 0.45). The X-40A first executes a steep glide scope, transitions to a shallower glide slope and then performs an abrupt flare maneuver prior to touchdown, as shown in the figure 6 .
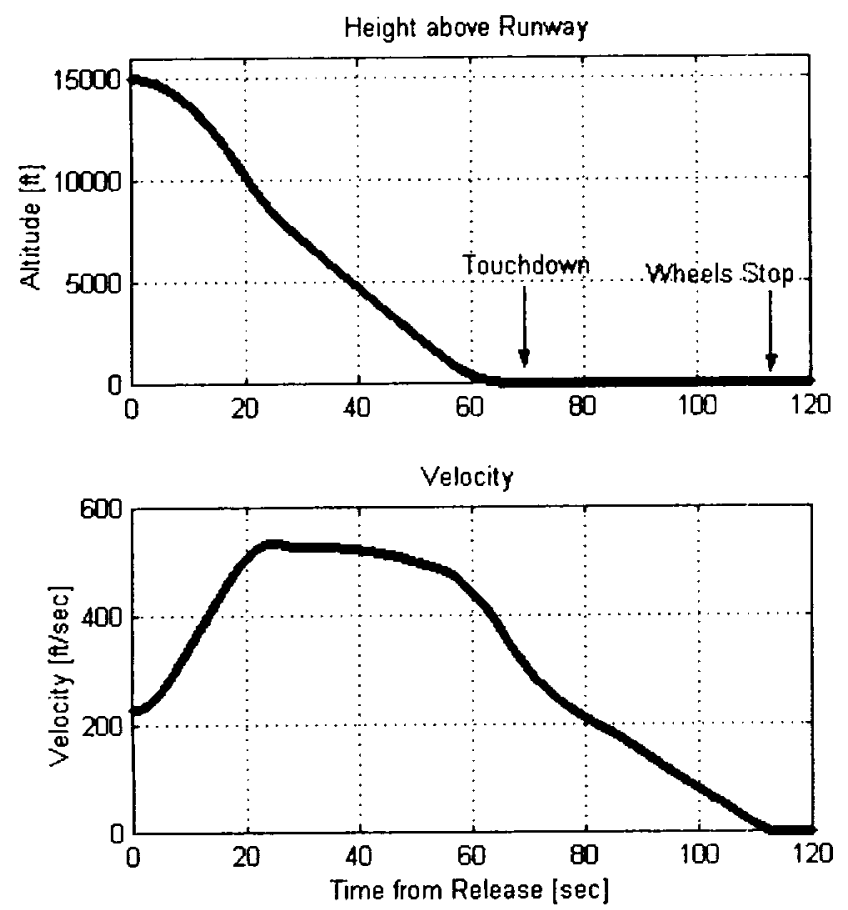

Figure 6. X-40A Free Flight Trajectory vs. Time

\section{Test results}

Telemetry data were collected for over 100 hours of SIGI testing with over 40 hours during approach or landing conditions. The focus of this report is on the drop phase of testing; from release to end of roll out.

During X-40A testing the SIGI gained a welldeserved, excellent reputation. The test conductors soon concluded that the SIGI was accurate and reliable. Although it was riding along as an experiment, it soon became the de facto truth source used to determine how well the CMIGITS and RAJPO were performing. This is not surprising given the high quality inertial instruments and 12 channel GPS receiver. Nevertheless, the results presented here compare the SIGI with the RAJPO TSPI.

The RAJPO performance was actually quite good, as one would expect from an L1/L2, keyed $Y$ code dGPS system, but it was never completely reliable until the RAJPO unit was properly aligned. That generally required substantial motion of the vehicle, because of the quality of the inertial package. There were a number of times in which the RAJPO unit had difficulties and the SIGI was able to help resolve them. On the other hand the CMIGITS was fairly reliable, but was less accurate, because of its lower quality inertial instruments and only five channel SPS GPS receiver. Five channels provide a higher PDOP than twelve and less robustness due to obscuration, which turned out to often be a problem.

The software to initialize and mode the SIGI required several iterations to be developed before the SIGI could be properly sequenced through alignment into navigation mode. It was verified during the static phase of testing. The minimum recommended align time was four minutes, but the $\mathrm{X}-40 \mathrm{~A}$ software included an alignment time of ten minutes to improve azimuth accuracy and contained provisions for up to 30 minutes for special testing.

Static navigation testing verified the performance of the SIGI and dGPS radio link and software. It was also used to characterize the RAJPO as a reference compared to SIGI. Figures 7 and 8 show stationary position and velocity errors, respectively. This data was collected after wheel stop on the runway to ensure data representative of a well aligned RAJPO. The $y$ axis scales are identical for the plots in Figure 8.

Dynamic test results for the blended solution proved difficult to compare to the truth reference, because of the free running relative time tag used. The creation of an absolute time tag was not entirely successful. Figure 9 shows horizontal position difference relative to the RAJPO during captive flight. The shifts and spikes of 10 to 15 feet can be attributed to shifts in the time tag generation scheme. The "true" SIGI performance is best characterized by the thread through the center of the plot. The shifts shown in the figure cannot be attributed to the RAJPO, because they were not present when comparing RAJPO to the SIGI's GPSonly solution or to the CMIGITS. 

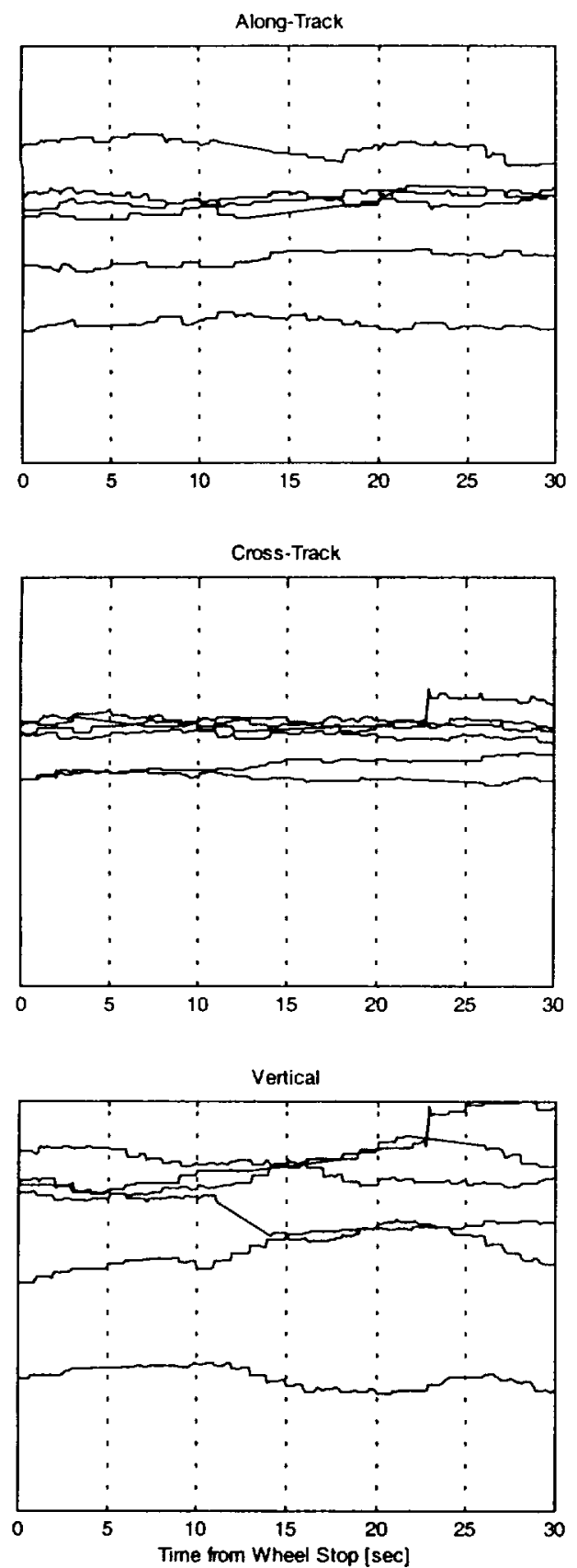

\section{Figure 7. SIGI - RAJPO stationary position accuracy}

It is straightforward, but time consuming to estimate and compensate for time shifts between the SIGI and the truth reference. However, a reliable, automatic method to handle the shifts has not yet been developed. Therefore, for this paper it was decided to concentrate on just a small portion of each flight test. The most important portion of the test in regard to the $\mathrm{X}-37$ program is the free flight phase--from release to rollout on the runway.
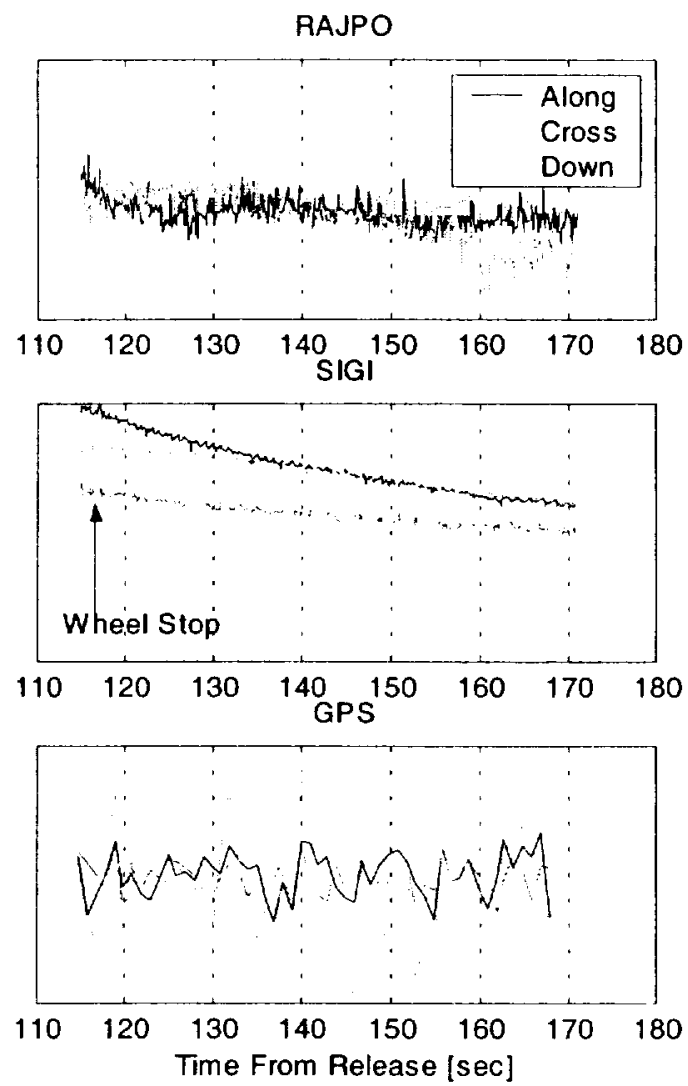

Figure 8. Comparison of RAJPO, SIGI and GPS stationary velocity error from Flight 7

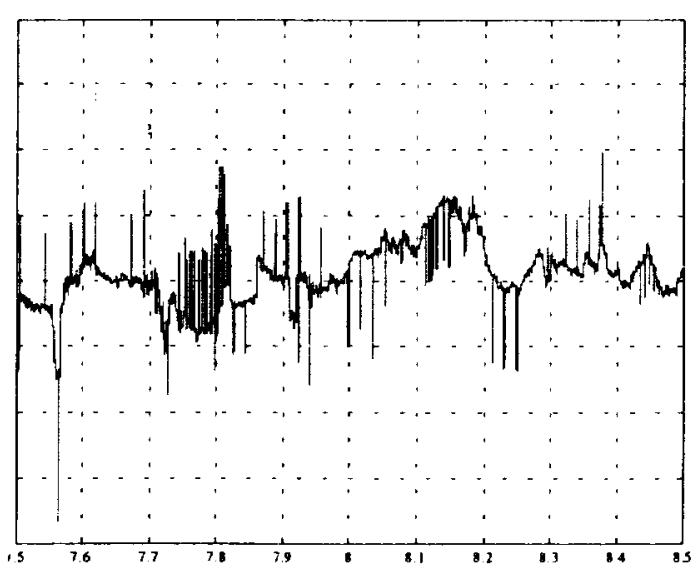

Figure 9. Position Difference (SIGI - RAJPO) during 1 hour of captive flight 


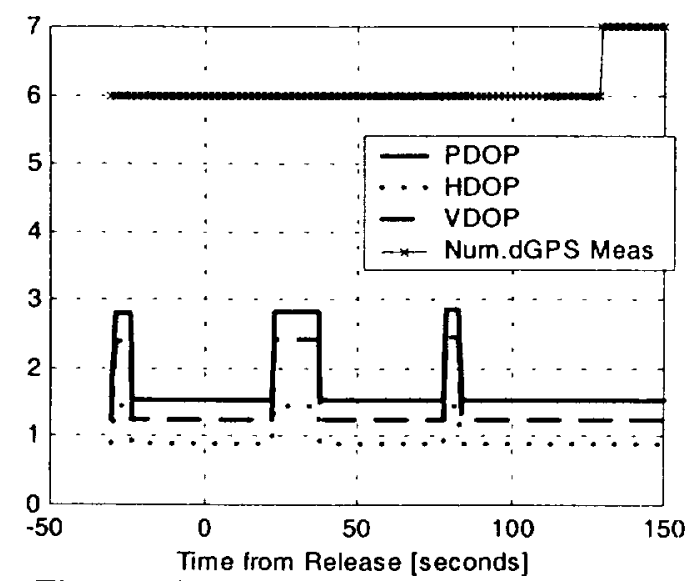

Figure 10. DOPS and Number of GPS Measurements for Flight 7

The GPS satellite geometry was similar for all seven X-40A free flights. Satellite tracking

SIGI minus RAJPO - Along Track Pos.Err
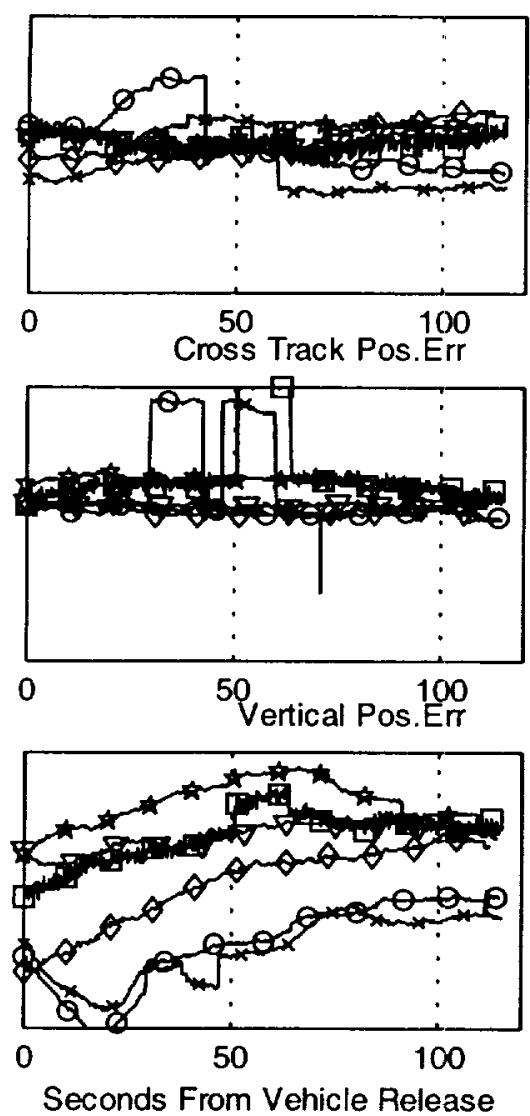

reliability by the CMIGITS during vehicle pitchover forced this arrangement of the test. Figure 10 shows the DOPS and number of satellites as output by SIGI during flight seven and is typical of all flights. The shifts are due to satellite constellation changes due to vehicle attitude maneuvers.

The blended and GPS solutions were compared to RAJPO truth reference during the seven free flights. The difference between the navigation solutions of the two systems are composed of SIGI and RAJPO position errors. The RAJPO TSPI errors during the free flights are specified to be much smaller than SIGI's (see Figure 7), so it is assumed that the plotted differences correspond mostly to SIGI errors.

GPS minus RAJPO -- Along Track Pos.Err
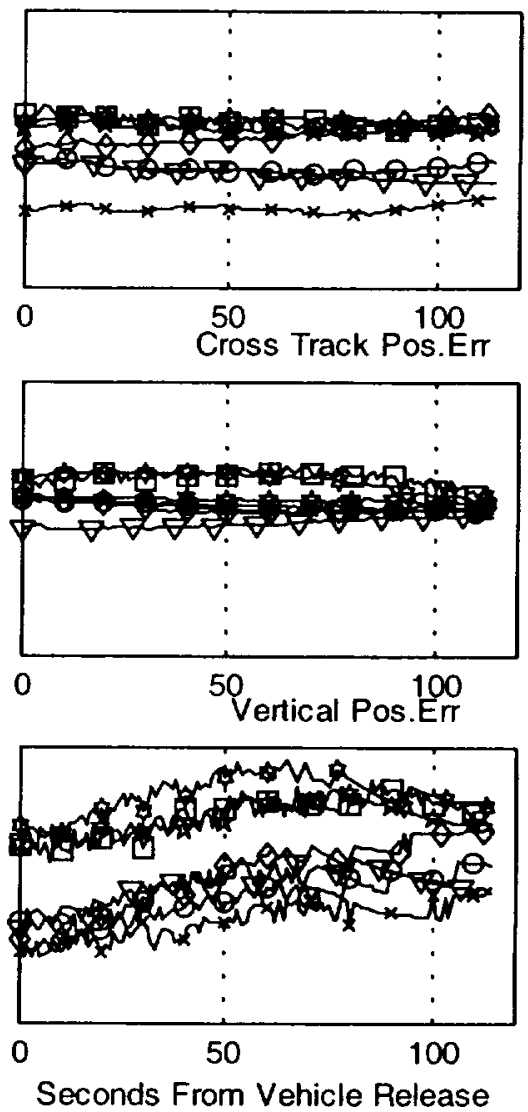

Figure 11. Position differences (SIGI - RAJPO) and (GPS - RAJPO) for flight test ensemble 
SIGI minus RAJPO -- Along Track Vel.Err

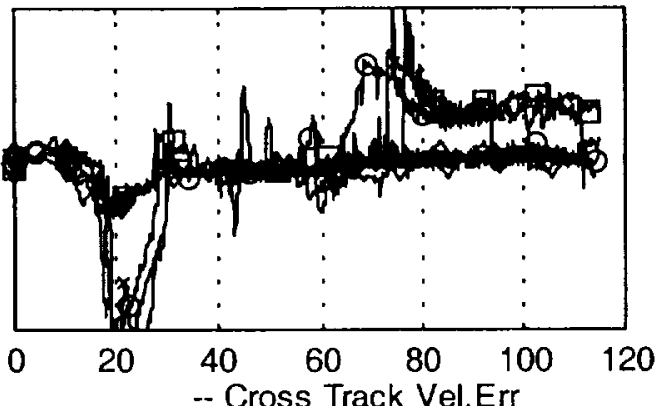

-- Cross Track Vel.Err
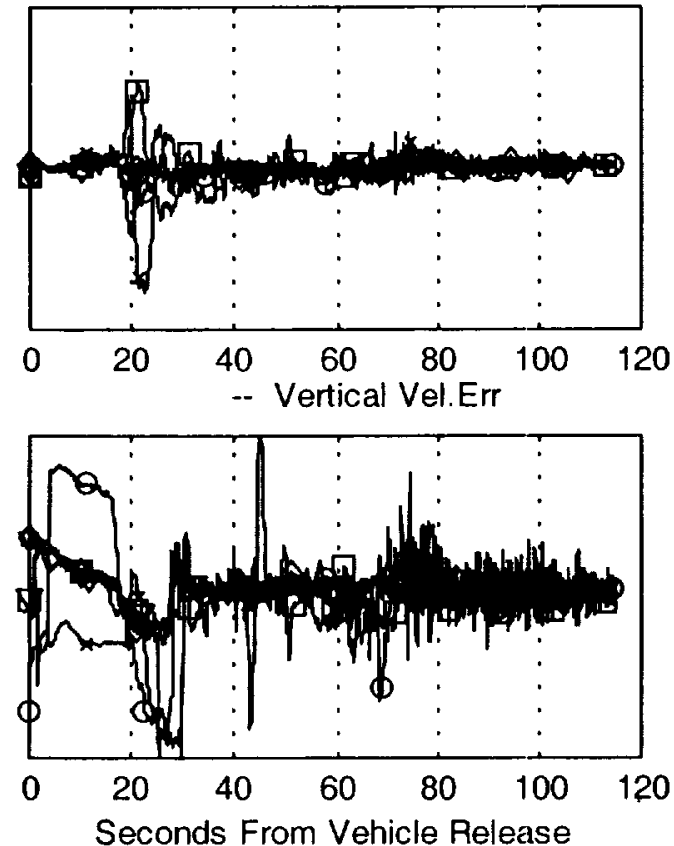

GPS minus RAJPO Along Track Vel.Err
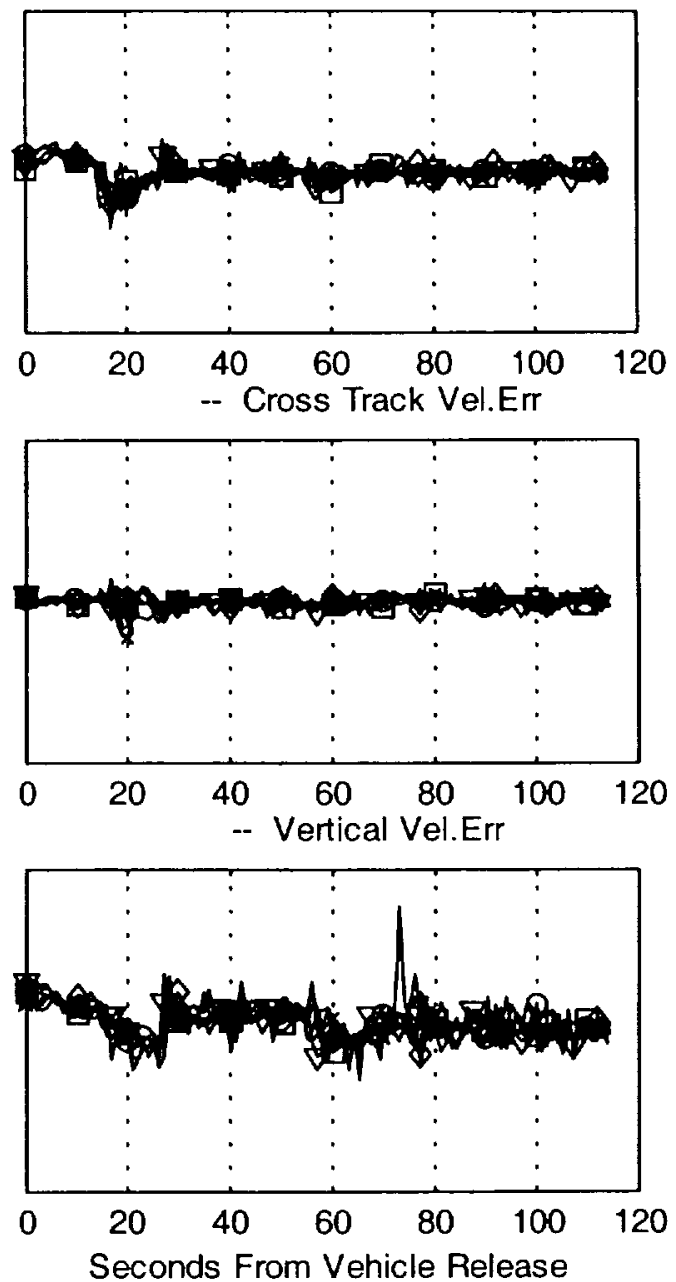

Figure 12. Velocity differences (SIGI - RAJPO) and (GPS - RAJPO) for flight test ensemble

The position differences are plotted in Figure 11 in runway relative coordinates (along track, cross track and vertical). The blended solution errors are shown in the three plots on the left and the GPS solution errors are shown in the three plots on the right. Some evidence remains of time tag errors in the blended solution. In fact the fifth flight was not included in the blended solution plots, because the time tag error is still unresolved. However, the GPS-only solution shows no such problem. Comparing the blended and GPS errors shows that in general the blended solution errors are correlated with the GPS errors.
The velocity differences are plotted in Figure 12 in similar fashion to position. Here the residual time tag errors are much more evident than in the position error plots, especially in the along and vertical axes.

The position errors from the flight tests were used to create ensemble statistics to compare to the X-37 SIGI specification. The SIGI specs for position error have been corrected to account for the actual GPS SV geometry during the flight test. 
SIGI minus RAJPO Position Error Statistics

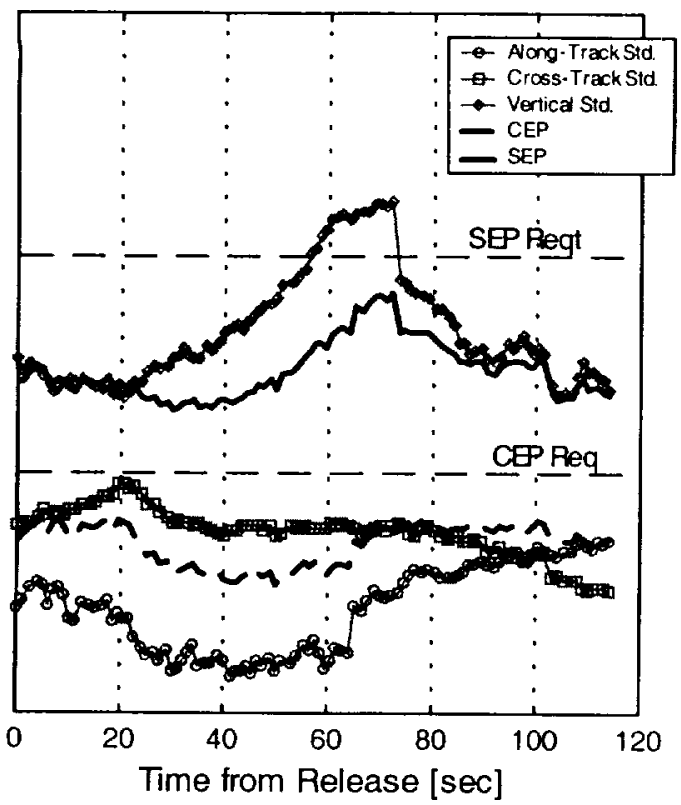

Figure 13. Ensemble Statistics for SIGI Solution

The statistics for the flight test ensemble are shown in Figures 13 (SIGI blended) and 14 (GPSonly) as compared to the X-37 requirements. Again, flight 5 was not included in the blended ensemble results. The plots show that the SIGI solutions are below the SEP and CEP requirements by several feet over the entire free flight and roll out phase.

GPS minus RAJPO Position Error Statistics

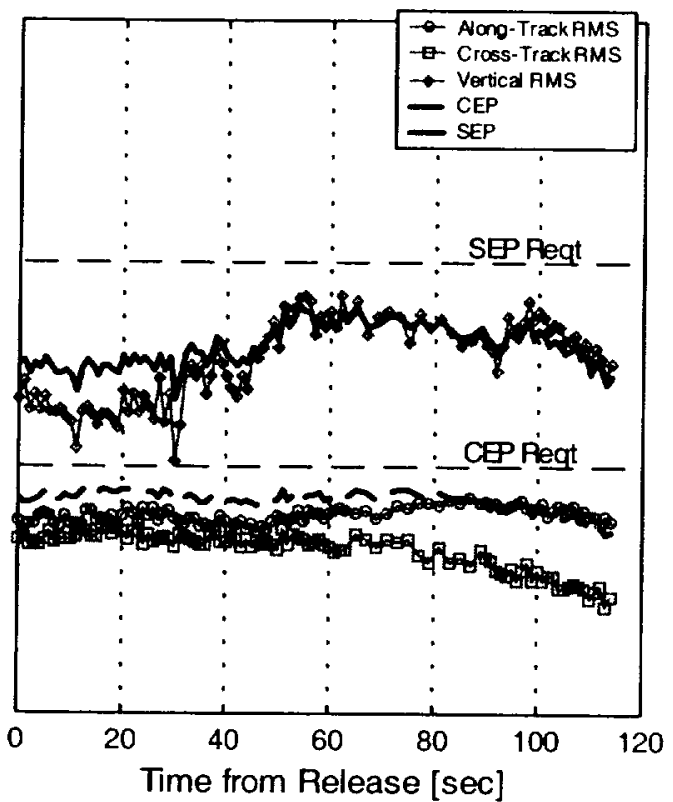

Figure 14. Ensemble Statistics for GPS-only Solution
Figure 15 shows a typical plot of SIGI blended Kalman filter residuals. The residual were a very useful aid to monitor during X-40A testing to provide a measure of assurance that the SIGI was behaving properly and that dGPS was being incorporated. The figure dramatically shows the advantage of dGPS. There is a short period in the center in which dGPS corrections were not available to the SIGI, because of obscuration of the receiving antenna. For this brief example the RMS of the residuals is about three times smaller (better) during dGPS periods than during periods without dGPS corrections.

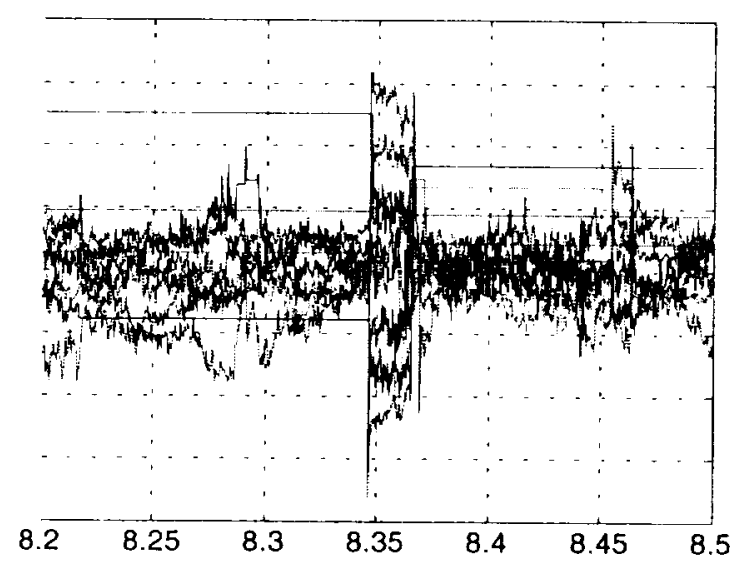

Figure 15. SIGI blended Kalman filter residuals

SIGI reliability during the $\mathrm{X}-40 \mathrm{~A}$ test program was excellent. There were no failures of SIGI over the entire course of testing. The SIGI operated in flight for over 15 hours (under the helicopter or in free flight), over 25 hours on the ground preflight and over 60 hours during ground and taxi testing. This test time is too short to make a meaningful reliability estimate for the $\mathrm{X}-\mathbf{3 7}$ mission. For example based on no failures and for the given test time the resulting flight environment MTBF is only 808 minutes at the $90 \%$ confidence level. Much more test time would be required to verify the spec MTBF, which is $6,500 \mathrm{hrs}$ for an uninhabited fighter environment.

\section{Conclusion}

Based on the approach and landing testing of the $\mathrm{X}-40 \mathrm{~A}$ the $\mathrm{X}-37$ SIGI confirmed reliable and accurate performance. Initialization, data and power 
interface, and dGPS operation were all demonstrated. The X-37 SIGI software interface is currently being developed using the experience gained from X-40A.

The blended solution performance was inferred to meet the X-37 requirements after correction of timing errors and comparison to the GPS-only solution's performance. Unfortunately, its performance could not be thoroughly verified, due to lack of an absolute time reference for it.

The GPS and dGPS performance of the Force 5 receiver was solid throughout testing. The twelve channel receiver never experienced less than four satellites throughout testing. Unlike the five channel receiver in the CMIGITS, it maintained an accurate navigation solution at all times, even during the severe pitch-over of the vehicle on release. This makes mission planning much more simple.

The lessons learned from X-40A testing of the SIGI that are applicable to X-37:

1. An absolute time reference, such as GPS or UTC is essential for verification and characterization of all navigation solutions of interest to the flight GN\&C. Even if an absolute time will not be used by the real time flight software, it is required for comparison of the navigation data to an on board truth reference during dynamic flight testing. The X-37 SIGI will have such a time reference.

2. A twelve channel receiver, as in the Force 5, is required for reliable test performance under all conditions.

3. Confidence in the SIGI's navigation performance will allow a direct mapping of the SIGI parameters to replace CMIGIT's for X-37 GN\&C.

4. SIGI data from the $\mathrm{X}-40 \mathrm{~A}$ flight test program contain a wealth of information for the X-37 program and should continue to be analyzed for characterization and modeling of the X-37 SIGI.

\section{Acknowledgements}

The authors would like to acknowledge the hard work and dedication of Mark Todd at Boeing for his contributions to this paper without whom this would not have been completed.
Much of this work was performed under the NCC8-190, Future-X/Pathfinder Flight Demonstrator cooperative agreement with NASA/Marshal Space Flight Center.

\section{References}

(1) Willms, Bob, "Space Integrated GPS/INS (SIGI) Navigation System for Space Shuttle", IEEE 1999 